\title{
Strates
}

STRATES Matériaux pour la recherche en sciences sociales

$15 \mid 2008$

Union européenne - Voisinages. La quête d'une intégration régionale

\section{Les pays méditerranéens et le marché mondial des produits agroalimentaires : le cas des fruits et légumes ou l'impératif de la solidarité}

\author{
Jean-Louis Rastoin
}

\section{CpenEdition}

Journals

Édition électronique

URL : http://journals.openedition.org/strates/6536

DOI : 10.4000/strates.6536

ISSN : 1777-5442

Éditeur

Laboratoire Ladyss

Édition imprimée

Date de publication : 1 janvier 2008

Pagination : 97-106

ISSN : 0768-8067

Référence électronique

Jean-Louis Rastoin, "Les pays méditerranéens et le marché mondial des produits agroalimentaires : le cas des fruits et légumes ou l'impératif de la solidarité ", Strates [En ligne], 15 | 2008, mis en ligne le 22 janvier 2013, consulté le 08 septembre 2020. URL : http://journals.openedition.org/strates/6536 ; DOI : https://doi.org/10.4000/strates.6536

Ce document a été généré automatiquement le 8 septembre 2020

Tous droits réservés 


\title{
Les pays méditerranéens et le marché mondial des produits agroalimentaires : le cas des fruits et légumes ou l'impératif de la solidarité
}

\author{
Jean-Louis Rastoin
}

\section{NOTE DE L'AUTEUR}

Ce texte reprend la communication présentée à au Conseil régional de Provence-AlpesCôte d'Azur dans le cadre du colloque de l'IPEMed du 5 juillet 2007 sur l'agriculture euroméditerranéenne.

1 L'objectif de ce texte est de montrer les enjeux régionaux et globaux d'un secteur d'activité déterminant pour l'espace euroméditerranéen ${ }^{1}$ : les fruits et légumes. On le sait, ses trois sous-ensembles révèlent de fortes disparités : les PSEM (Pays du sud et de l'est de la Méditerranée), $56 \%$ des habitants, ne réalisent que $13 \%$ du PIB de la zone ; pour l'Est adriatique (Croatie, Serbie Monténégro, Albanie), les chiffres sont 4 et $1 \%$ et, pour les pays méditerranéens de l'Union européenne (« UE-Med»), 4 et $86 \%$. Les écarts se creusent encore si l'on considère le revenu par tête (de 1 à 7 en parités de pouvoir d'achat), la productivité totale du travail (de 1 à 15), la productivité agricole (de 1 à 20). En revanche, la croissance économique est deux fois plus élevée dans les PSEM (environ $5 \%$ par an sur 1994-2004) que dans les pays de l'UE.

On note encore dans les PSEM un poids considérable de l'emploi agricole : près de 33 millions d'actifs et $34 \%$ de la population active totale, contre 5 millions et $6 \%$ dans l'UE-Med, les pays de l'Est Adriatique se situant entre les deux. La dépendance extérieure pour l'alimentation se situe à un niveau très élevé dans les PSEM, avec un 
déficit commercial dépassant 5 milliards de dollars en moyenne 2002-2004, face à un énorme excédent de la rive nord de la Méditerranée (29 milliards de dollars).

3 Ces constatations appellent une analyse que l'on structurera autour de trois points : la nécessité d'identifier les produits stratégiques de l'agroalimentaire en Méditerranée ; la valorisation de ces produits sur le marché international ; les performances des filières méditerranéennes dans un contexte de globalisation des marchés. On conclura par les implications politiques et stratégiques du diagnostic établi.

\section{Huit produits agroalimentaires stratégiques pour la Méditerranée}

4 L'analyse présentée ici concerne huit produits végétaux : les agrumes, les autres fruits, les pommes de terre, les tomates, les autres légumes, l'huile d'olive, les olives de table et le blé. Si les fruits et légumes constituent une ressource essentielle à l'exportation pour l'agriculture des pays méditerranéens, le blé reste une denrée alimentaire de base essentielle pour ces pays et en général déficitaire, ce qui légitime la liste des produits retenus. Pour chacun de ces produits, on a établi quatre groupes d'indicateurs en vue de procéder à un benchmarking intra et extra-régional : la production en volumes, le commerce extérieur (importations et exportations) en volumes, les prix internationaux et enfin les ratios de performance.

5 La région euroméditerranéenne présente une indéniable spécialisation dans la production végétale. Pour les huit produits considérés, à l'exception des pommes de terre et des autres légumes, elle obtient, pour la période 2002-2004, un score supérieur à celui de son PIB, si l'on considère sa part dans le total mondial. Ce score s'établit à $99 \%$ pour l'huile d'olive, à $9 \%$ pour la pomme de terre, en passant par des valeurs élevées pour la tomate (31\%), et moyennes pour les agrumes (19\%), les autres fruits et le blé ( $16 \%)$, les autres légumes ( $12 \%)$. Cependant, les tendances dans les dix dernières années sont déclinantes pour tous les produits sauf le blé et la pomme de terre (stagnation). Une analyse plus fine montre que ce déclin concerne les pays méditerranéens de l'UE. En effet, la part de la production des PSEM au sein des 22 pays Euromed, qui est de 20 à $40 \%$ selon les produits, progresse, à l'exception de l'huile d'olive du fait de la montée en puissance de la production espagnole.

6 Les exportations des huit produits par l'Euromed représentent une fraction du total mondial généralement supérieure à celle occupée par la production, ce qui traduit une extraversion des filières considérées. Cela va de $15 \%$ des exportations mondiales de blé à 25-30\% pour les autres fruits et légumes et à $52 \%$ pour les agrumes. Mais contrairement à ce qui est observé pour la production, les PSEM n'exportent qu'une part modeste de leurs produits. Le cas extrême est celui de l'huile d'olive : $9 \%$ de la production des PSEM est exportée, alors que pour les pays de l'UE-Med la proportion atteint $90 \%$. On peut donc faire une double hypothèse : les PSEM disposent d'un secteur d'exportation contraint par le marché intérieur; le potentiel de croissance des exportations des PSEM est ouvert mais conditionné par les ressources mobilisables (terre, eau, financements et capacité d'organisation).

7 La demande adressée aux huit produits agricoles retenus est composée de la consommation nationale et des importations. Les marchés intérieurs les plus importants se situent dans les pays les plus peuplés, s'agissant de produits de base du 
régime alimentaire. Nous avons considéré cinq grandes zones dans le monde. Le « reste du monde " est une catégorie hétéroclite qui rassemble les autres pays que ceux faisant partie de grandes unions économiques (UE, Alena, Mercosur) et la Grande Chine (Chine et Honk Kong). Pour la plupart des produits, le reste du monde se situe au premier rang pour la consommation apparente (production + importations - exportations), avec 30 à $40 \%$ de la demande mondiale, suivie par l'Ue à 25 (de 10 à $20 \%$ ). Deux produits occupent une place spécifique : l'huile d'olive, dont $74 \%$ de la demande émane de l'UE à 25 ; les agrumes, avec $21 \%$ de la consommation apparente dans le Mercosur, $19 \%$ dans l'Alena et seulement11 \% dans l'UE à 25. De 1994 à 2004, la croissance la plus forte de la demande domestique se situe en Chine (de 60 à $600 \%$, à l'exception du blé qui régresse, tabl. 1), ce qui confirme l'émergence d'un très grand marché, avec un phénomène simultané qui est celui, signalé plus haut, de l'explosion des exportations. L'UE à 25 reste dynamique du fait principalement des nouveaux États membres. Enfin, on a ici la confirmation de l'importance du marché intérieur pour les PSEM.

Tableau 1 : Dynamique de la consommation apparente de huit produits agricoles.

\begin{tabular}{|c|c|c|c|c|c|c|c|}
\hline $\begin{array}{lr}\text { Évolution } & \text { en } \\
\text { volume } & (\% \\
1992-94 & \text { à } \\
2002-04) & \end{array}$ & $\begin{array}{l}\text { EU } \\
25\end{array}$ & PSEM & ALENA & Mercosur & Chine & $\begin{array}{c}\text { Reste } \\
\text { du } \\
\text { monde }\end{array}$ & Monde \\
\hline Agrumes & 13 & 22 & 27 & 3 & 109 & 27 & 25 \\
\hline Autres fruits & -1 & 22 & 16 & 6 & 142 & 24 & 27 \\
\hline $\begin{array}{l}\text { Pommes de } \\
\text { terre }\end{array}$ & $\begin{array}{c}- \\
18\end{array}$ & 37 & 13 & 13 & 62 & 16 & 14 \\
\hline Tomates & 18 & 48 & 10 & 33 & 166 & 41 & 49 \\
\hline $\begin{array}{l}\text { Autres } \\
\text { légumes }\end{array}$ & 18 & 42 & 20 & 37 & 141 & 32 & 66 \\
\hline Huile d'olive & 49 & 17 & 98 & 17 & 635 & 73 & 46 \\
\hline Olives & 56 & 49 & -9 & 30 & 1 & 127 & 53 \\
\hline Blé & 39 & 29 & 0 & 26 & -17 & 3 & 7 \\
\hline
\end{tabular}

Source : Faostat, 2006

8 L'UE à 25 reste le premier marché mondial pour les échanges extérieurs, avec 25 à $60 \%$ des importations mondiales pour les huit produits analysés. Les tendances sur dix ans sont à la hausse pour l'ensemble des produits, notamment pour les tomates, l'huile d'olive et les autres légumes.

9 La dépendance extérieure des PSEM est quasiment nulle pour sept produits : le ratio importations/consommation apparente est, en moyenne 2002-2004, de 1 à $5 \%$ pour les différents fruits et légumes et les produits de l'olivier, mais de $32 \%$ pour le blé. Pour les 
pays UE-Med, ce ratio s'établit entre 20 et $30 \%$ pour les fruits et légumes, à $40 \%$ pour l'huile d'olive et à $30 \%$ pour le blé.

\section{Une valorisation inégale des productions méditerranéennes}

La valorisation des produits est estimée à travers les prix apparents moyens à l'exportation (valeur des exportations/volumes exportés). Pour les agrumes, ils varient de 356 dollars la tonne dans le Mercosur à 710 dans l'UE-Med, avec 390 dans les PSEM. D'une manière générale, les PSEM accusent une décote de leurs prix de 25 à $70 \%$ par rapport aux pays méditerranéens de l'Union européenne. Seul le blé est vendu plus cher (+ $3 \%)$, mais pour de faibles quantités.

11 L'UE à 25 est la région qui consent les prix les plus élevés à l'importation : $11 \%$ à $30 \%$ de plus que le prix moyen mondial pour ces fruits et légumes, sauf pour la pomme de terre (prix identique) et l'huile d'olive (-4 \% par rapport au prix mondial, 2002-2004).

On en tire deux conclusions : d'une part les PSEM ne parviennent pas à valoriser leurs produits aussi bien que leurs concurrents de la rive nord de la Méditerranée ; d'autre part le commerce de proximité est rémunérateur puisque l'UE-25 importe ses produits à des tarifs plus élevés que les autres grands importateurs mondiaux (Alena et Chine notamment).

13 Le tableau 2 permet de mesurer l'importance des marchés sur lesquels les pays méditerranéens sont présents, avec des produits correspondant à leur vocation agroclimatique. Pour les huit produits retenus les exportations mondiales dépassaient, en moyenne 2002-2004, 90 milliards de dollars, soit $18 \%$ de l'ensemble des produits agricoles et alimentaires. Les secteurs caractéristiques du commerce méditerranéen figurent parmi les plus importants : les fruits et légumes au sens large totalisent plus de 77 milliards. La part de marché mondial des pays de l'UE à 25 est proche de $50 \%$, cependant il s'agit quasi exclusivement d'exportations intra-zone. Les PSEM ne représentent au maximum que $12 \%$ des exportations mondiales en valeur. En additionnant les exportations de l'UE vers les pays tiers et celles des PSEM, la part de marché mondial de cet ensemble Europe + Méditerranée (36 pays) se situe à $13 \%$ pour les huit produits analysés, avec un pic à $44 \%$ pour l'huile d'olive. Cela peut être interprété comme une sous-valorisation des produits méditerranéens et en particulier des PSEM sur le marché international.

Le marché des importations de l'UE à 25 pour sept des huit produits retenus (le blé est ici exclu) représentait en 2002-2004 près de 44 milliards de dollars, dont $77 \%$ étaient constitués de commerce intra-régional. Les importations en provenance des pays tiers se montaient à 10 milliards de dollars, dont 3 milliards originaires des PSEM. Autrement dit, les PSEM représentent à peine $7 \%$ des approvisionnements externes en fruits et légumes des 25 pays de l'UE et $29 \%$ si l'on ne prend en compte que les importations extra-communautaires. 
Tableau 2 : Poids de la région méditerranéenne dans les exportations mondiales, moyenne 2002-2004.

\begin{tabular}{|l|c|c|c|c|}
\hline Produits & $\begin{array}{c}\text { Total } \\
\text { monde } \\
\text { (M \$) }\end{array}$ & $\begin{array}{c}\text { Part de } \\
\text { marché UE } \\
\text { à 25 }\end{array}$ & $\begin{array}{c}\text { Part de } \\
\text { marché } \\
\text { PSEM (11) }\end{array}$ & $\begin{array}{c}\text { Part de } \\
\text { marché extra } \\
\text { UE + PSEM }\end{array}$ \\
\hline $\begin{array}{l}\text { Autres } \\
\text { fruits }\end{array}$ & 35563 & $42 \%$ & $6 \%$ & $11 \%$ \\
\hline $\begin{array}{l}\text { Autres } \\
\text { légumes }\end{array}$ & 26405 & $53 \%$ & $5 \%$ & $11 \%$ \\
\hline Blé & 16608 & $26 \%$ & $1 \%$ & $10 \%$ \\
\hline Agrumes & 5818 & $58 \%$ & $11 \%$ & $18 \%$ \\
\hline Tomates & 4103 & $60 \%$ & $9 \%$ & $13 \%$ \\
\hline $\begin{array}{l}\text { Huile } \\
\text { d'olive }\end{array}$ & 3323 & $87 \%$ & $12 \%$ & $44 \%$ \\
\hline $\begin{array}{l}\text { Pommes } \\
\text { de terre }\end{array}$ & 1931 & $75 \%$ & $8 \%$ & $24 \%$ \\
\hline Olives & 55 & $73 \%$ & $1 \%$ & $10 \%$ \\
\hline Total & 93806 & $46 \%$ & $6 \%$ & $13 \%$ \\
\hline
\end{tabular}

Source : Calculs de l'auteur sur données Comtrade, 2006.

Tableau 3 : Importations de fruits et légumes l'UE à 25,moyenne 2002-2004.

\begin{tabular}{|l|c|c|c|c|}
\hline Produits & $\begin{array}{c}\text { Import } \\
\text { EU-25 (M \$) }\end{array}$ & $\begin{array}{c}\text { Extra UE/ } \\
\text { Total UE }\end{array}$ & $\begin{array}{c}\text { PSEM/ } \\
\text { Total EU }\end{array}$ & $\begin{array}{c}\text { PSEM/ } \\
\text { Extra UE }\end{array}$ \\
\hline Autres fruits & 20034 & $34 \%$ & $7 \%$ & $21 \%$ \\
\hline $\begin{array}{l}\text { Autres } \\
\text { légumes }\end{array}$ & 14204 & $14 \%$ & $5 \%$ & $35 \%$ \\
\hline Agrumes & 3664 & $19 \%$ & $7 \%$ & $37 \%$ \\
\hline Tomates & 2441 & $7 \%$ & $6 \%$ & $93 \%$ \\
\hline Huile d'olive & 2149 & $14 \%$ & $14 \%$ & $99 \%$ \\
\hline $\begin{array}{l}\text { Pommes de } \\
\text { terre }\end{array}$ & 1258 & $9 \%$ & $8 \%$ & $98 \%$ \\
\hline
\end{tabular}




\begin{tabular}{|l|c|c|c|c|}
\hline Olives & 37 & $5 \%$ & $1 \%$ & $10 \%$ \\
\hline Total & 43787 & $23 \%$ & $7 \%$ & $29 \%$ \\
\hline
\end{tabular}

Source : Comtrade, 2006

15 L'UE à 25 exporte des produits agricoles, principalement laitiers et céréaliers, vers les PSEM pour des montants comparables aux importations agricoles réalisées en provenance de ces pays. Les exportations agricoles de l'UE vers les PSEM représentent $4 \%$ de l'ensemble des destinations, les importations agricoles de l'UE originaires des PSEM représentent $3 \%$ des importations agricoles totales de l'UE. L'enjeu agricole des discussions de l'accord de libre-échange euroméditerranéen est donc limité pour l'UE. Il est en revanche important pour les PSEM : 34 \% des exportations agricoles des PSEM dépendent des fruits et légumes, pour l'essentiel dirigés vers l'UE ; mais compte tenu de la modicité des exportations agricoles de ces pays, cela ne porte que sur des montants limités, environ 3,5 milliards de dollars. Pour les huit pays méditerranéens membres de l'UE, dont les flux sont également dirigés principalement vers le marché européen, le ratio n'est que de $25 \%$ mais cela représente des sommes bien plus considérables ( 22 milliards). La concurrence intraméditerranéenne est donc largement surestimée par les organisations professionnelles.

\section{Benchmarking international des productions méditerranéennes}

Un score synthétique a été établi pour les fruits et légumes (six produits). Ce score mesure la performance de chaque pays et pour chaque produit en prenant en compte l'importance du secteur sur le marché mondial, sa dynamique sur dix ans, la valorisation des produits et enfin la spécialisation internationale relative.

Ce score met en évidence la suprématie espagnole, à la fois en termes de taille critique (leadership des exportations) et de performance, Les États-Unis arrivent en seconde position et l'Italie en troisième. On relèvera la croissance rapide du secteur à Chypre, en Syrie et en Turquie, ainsi que la 11` place occupée par le Maroc dont les exportations pourraient atteindre rapidement 1 milliard de dollars. La place des pays méditerranéens est cependant souvent menacée par les pays de l'Alena ou du Mercosur ou encore de la Chine et du Brésil. À noter que le Chili ne faisait pas partie de l'analyse, or on connait sa forte position sur le marché international des fruits et légumes.

Ce benchmarking place en $10^{\mathrm{e}}$ position la Turquie du fait de la méthode de calcul retenue. Il faut néanmoins insister sur le poids considérable et le dynamisme de ce pays sur l'échiquier agricole mondial et euro-méditerranéen. La Turquie disposait en 2003 de 40 millions d'hectares de surface agricole (187 millions dans l'UE à 25) et de plus de 7 millions d'actifs dans l'agriculture (autant que l'UE à 25), soit le tiers de l'emploi total. Le pays se situe au premier rang mondial pour la production de melons, de noisettes, d'abricots, de figues et de cerises. En 2006, la Turquie a exporté pour 8,6 milliards de dollars de produits agricoles, ce qui la situe au 27 rang mondial, mais son taux de croissance par rapport à 2000 atteint $126 \%$, en 3 position derrière le Brésil et l'Indonésie. La compétitivité internationale du pays est élevée pour les fruits dont elle a 
le leadership de la production, et certains légumes. En revanche, pour les céréales, les produits animaux et les produits agroalimentaires, les experts prévoient un déficit croissant dans le contexte d'une extension de l'union douanière avec l'UE, du fait de l'importance du marché intérieur (plus de 85 millions d'habitants en 2025), mais aussi un effet stimulant pour la modernisation du secteur à travers notamment l'amélioration du cadre institutionnel (Jacquet et Cakmak, 2005). Le cas de la Turquie est révélateur, à un niveau amplifié, de la situation actuelle et des perspectives d'évolution de la plupart des PSEM.

Tableau 4 : Benchmarking international des filières fruits et légumes, 2002-2004.

\begin{tabular}{|c|l|c|c|}
\hline & & Export (M \$) & Score global \\
\hline $\mathbf{1}$ & Espagne & 12599,0 & 6531 \\
\hline $\mathbf{2}$ & États-Unis & 5693,7 & 2928 \\
\hline $\mathbf{3}$ & Italie & 5328,5 & 2779 \\
\hline $\mathbf{4}$ & Chypre & 118,5 & 2576 \\
\hline $\mathbf{5}$ & Mexique & 4127,0 & 2244 \\
\hline $\mathbf{6}$ & France & 3037,5 & 1576 \\
\hline $\mathbf{7}$ & Chine & 1666,0 & 1325 \\
\hline $\mathbf{8}$ & Syrie & 200,7 & 935 \\
\hline $\mathbf{9}$ & Canada & 1604,6 & 928 \\
\hline $\mathbf{1 0}$ & Turquie & 1227,2 & 716 \\
\hline $\mathbf{1 1}$ & Maroc & 795,5 & 496 \\
\hline $\mathbf{1 5}$ & Argentine & 804,9 & 482 \\
\hline $\mathbf{1 3}$ & Israël & 518,0 & 462 \\
\hline $\mathbf{1 4}$ & Grèce & 854,2 & 449 \\
\hline $\mathbf{1 5}$ & Brésil & 366,4 & 237 \\
\hline
\end{tabular}




\begin{tabular}{||c|c|c|}
\hline Monde & 65728,9 & 33503 \\
\hline UE 25 & 25735,1 & 13203 \\
\hline EUROMED (22) & 36631,1 & 18750 \\
\hline UE-MED (8) & 23182,1 & 11898 \\
\hline PSEM (11) & 15390,7 & 7926 \\
\hline Est Adriatique (3) & 47,1 & 189 \\
\hline MERCOSUR & 3052,5 & 1592 \\
\hline ALENA & 1259,5 & 697 \\
\hline
\end{tabular}

Source : Rastoin, Szedlak, 2006

\section{Conclusion : l'urgence d'une politique méditerranéenne dans l'agroalimentaire}

Ce diagnostic met en relief plusieurs évolutions dans les dix dernières années :

20

- les filières fruits et légumes caractérisent les pays méditerranéens au même titre que les productions animales et les grandes cultures marquent l'Europe septentrionale. Cependant, cette spécificité est mise en question par un déclin dont les causes sont multiples : économiques, sociales, environnementales, managériales ;

21 - les pays méditerranéens ont globalement des difficultés à maintenir leur part de marché internationale; les PSEM se révèlent plus dynamiques que les pays de l'UE, mais valorisent deux fois moins bien leurs produits à l'exportation ;

22 - contrairement à une opinion répandue, les enjeux économiques des échanges transméditerranéens de fruits et légumes sont relativement peu importants par rapport au commerce total ;

23 - il existe une érosion des opérateurs céréaliers européens sur le marché des PSEM, résultant de la montée des fournisseurs des autres zones de production.

24 Ce diagnostic appelle une politique volontariste pour maintenir dans la zone méditerranéenne des productions qui sont très importantes pour les économies locales. Des recompositions spatiales Nord-Sud devraient pouvoir être organisées en faisant jouer les complémentarités de gamme sur des produits similaires qui restent encore typiques de la région méditerranéenne. Il existe au niveau mondial un fort potentiel de croissance des marchés des fruits et légumes du fait du déséquilibre caractérisant actuellement les modèles de consommation alimentaire dans la plupart des pays du monde, avec l'apparition de pathologies de grande ampleur (obésité, diabète, maladies cardio-vasculaires, cancers, etc.) et du caractère prophylactique de ces produits. Le modèle de consommation alimentaire méditerranéen constitue, selon les nutritionnistes, une excellente réponse à ces pathologies menaçantes. Cependant, ce potentiel est d'ores et déjà identifié par de grands pays agricoles (États-Unis, Australie, 
Chili, etc.) qui lancent de vastes programmes de développement de leurs productions de fruits et légumes et d'huile d'olive. Les pays méditerranéens, berceau de ce modèle alimentaire, risquent fort de se voir menacés sur ces marchés à forte croissance par les nouveaux pays producteurs, à l'instar de ce que l'on observe pour le vin.

Il devient urgent de mettre en place au niveau euroméditerranéen de tels programmes de développement des filières fruits et légumes, incluant les produits transformés comme l'huile d'olive, en promouvant la spécificité régionale sur la base d'une identification d'origine. Les avantages à tirer de tels programmes seraient considérables, car outre l'expansion sur le marché international, ils ne peuvent manquer d'impacter les économies locales par :

26 - la « reconquête" ou tout simplement l'accompagnement du marché intérieur. En effet, d'une part ce modèle alimentaire disparaît des pays méditerranéens sous la pression du modèle agro-industriel médicalisé porté par les grandes firmes multinationales de l'agroalimentaire; d'autre part le marché des PSEM va s'accroître considérablement dans les vingt ans à venir : la population de ces pays dépassera 340 millions d'habitants en 2025 ( $+31 \%$ par rapport à 2004) et le nombre d'urbains 235 millions (+ $63 \%)$;

27 - le modèle de production peut être orienté vers des filières intégrées et courtes respectant les impératifs du développement durable, par opposition aux filières longues du schéma agro-industriel dont le bilan énergétique et social est préoccupant ;

28 - la prise en compte de la complémentarité euroméditerranéenne dans le domaine agroalimentaire (potentiel céréalier et bovin de l'UE, potentiel horto-fruticole des pays méditerranéens).

29 Quelles sont les contraintes à étudier et à lever? Ce sont principalement le déficit de volonté politique de coopération ; la fragilité des ressources naturelles (terre et eau); le manque de compétences techniques et managériales (notamment la capacité d'organisation des entreprises et des filières); le cadre institutionnel, souvent défaillant dans les PSEM; les blocages psychologiques et entrepreneuriaux, au Nord, comme au Sud de la Méditerranée.

30 La levée des contraintes comme la promotion des produits méditerranéens devraient relever d'une Politique agroalimentaire commune euroméditerranéenne (Pacem) qui s'appuierait sur les principes qui ont fait le succès de la PAC en les actualisant pour tenir compte de la nouvelle donne internationale (développement durable), et d'une spécificité typiquement méditerranéenne: l'ancrage territorial des produits alimentaires. Simultanément, il est nécessaire de faire évoluer la connaissance mutuelle des opérateurs privés des deux rives de la Méditerranée pour passer d'une logique de confrontation à une logique de partenariat. 


\section{BIBLIOGRAPHIE}

Allaya M., Pradeilles M. N., Ferrier I., 2006, Médagri, Annuaire des économies agricoles et alimentaires des pays méditerranéens et arabes, Ciheam-Iam, Montpellier.

Atlaséco, 2006, Atlas Économique Mondial 2007, Le Nouvel Observateur.

Emlinger, Ch., Jacquet, F., Petit, M., 2006, Les enjeux de la libéralisation agricole dans la zone méditerranéenne, Région et développement, $\mathrm{n}^{\circ} 23$.

Imbert E., 2002, Panorama du marché mondial des agrumes : l'évolution singulière des petits agrumes, Cirad-flore Journées Professionnelles, Montpellier, France, 10 au 10 octobre.

Jacquet F., Cakmak E., 2005, « Adhésion de la Turquie à l'Union européenne : un élargissement agricole plus délicat que les précédents ?», Déméter 2006, p. 9-56.

Lipchitz A., 2005, « La libéralisation agricole en zone euroméditerranée : la nécessité d'une approche progressive ", Notes et Études Économiques, $n^{\circ}$ 23, p. 113-156

Ould Aoudia J., 2006, Croissance et réformes dans les pays arabes et méditerranéens, AFD, Paris.

Radwan S., Reiffers J.-L., 2003, « L'impact de la libéralisation dans le contexte du partenariat euro-méditerranéen », Rapport Femise, Marseille, Institut de la Méditerranée.

Rastoin J.-L., Szedlak A., 2006, Dynamique des échanges internationaux agricoles et agroalimentaires de la zone Euro-méditerranéenne, Montpellier, IPEMed, UMR Moisa.

Rastoin J.-L., Ayadi N., Montigaud J.-C., 2006, « La vulnérabilité des régions européennes productrices de fruits et légumes frais et transformés dans un contexte de libéralisation internationale », Rapport de synthèse WP-2, 6th FPR, Impacts of agricultural trade liberalization between the UE and Mediterranean countries, [contrat SSPE-CT-2004-502457], Montpellier, UMR Moisa.

Regmi A., Ballenger N., Putnam J., 2004, « Globalisation and Income Growth Promote the Mediterranean Diet », Public Health Nutrition, vol. 7, nº 7, p. 977-983.

Roux B., 2006, Les PSEM et les échanges agricoles internationaux : vers une régulation euroméditerranéenne ou vers un libre échange globalisé ? Colloque International, Université Galatasaray, Istanbul, Turquie, 26 et 27 mai 2006.

Spreen T. H., 2001, Projections de la production et de la consommation mondiales d'agrumes en 2010, Symposium sur les agrumes, Chine/FAO.

\section{Sites Internet}

www.fao.org (FAOSTAT)

www.unstats.un.org (COMTRADE)

www.fruits-et-legumes.net (CTIFL - Centres techniques industriels de fruits et de légumes)

www.producteursdepommesdeterre.org (UNPT - Union nationale des producteurs de pommes de terre) 


\section{NOTES}

1. Cet espace est formé des pays riverains de la mer Méditerranée. En toute rigueur, la BosnieHerzégovine fait partie de la zone, en raison du corridor la reliant à la Méditerranée; nous ne l'avons cependant pas incluse ici.

\section{RÉSUMÉS}

L'article établit le diagnostic de l'agriculture méditerranéenne, notamment dans les fruits et légumes qui sont stratégiques pour l'agriculture de cette région, et montre les risques d'une libéralisation «sèche » des échanges agricoles dans le cadre de la zone de libre-échange prévue en 2010 dans l'espace Euromed. L'agriculture méditerranéenne est en pleine incertitude : la possible disparition de la PAC après 2013 obscurcit l'horizon de l'agriculture de l'Europe méditerranéenne, qui bénéficiait déjà bien peu de ses subventions; la concurrence s'intensifie entre rive nord et rive sud, et même entre pays du Sud; au Sud en général, la faiblesse de la productivité agricole fait craindre une déstabilisation majeure à mesure que la libéralisation des échanges agricoles accélérera l'exode rural. Enfin, et même si la prise de conscience n'en est pas encore claire du fait du repliement des préoccupations des différents agriculteurs de la région, la mondialisation des échanges agricoles fait arriver en Europe des concurrents de plus en plus lointains, y compris pour des produits «méditerranéens» typiques. Face à la dégradation des positions commerciales internationales des agricultures méditerranéennes de la rive nord comme de la rive sud, l'auteur en appelle à la définition d'une politique commune.

This paper draws the balance sheet of the Mediterranean agriculture. It is focused on the production of fruits and vegetables which is strategic in the economic structure of this region. It puts the stress on the danger of a brutal liberalisation of trade exchanges in the framework of the future Euromediterranean free trade area (2010). The Mediterranean agriculture is in a period of uncertainty: the possible end of the common agricultural policy in 2013 darkens the future of the agriculture of the Mediterranean regions of the European Union; the competition between the north and the south banks of the Mediterranean is growing, even between the countries of the Southern bank; on the South bank, the agricultural sector, which is already weak because of its low productivity, will be destabilized by the liberalisation of the commercial exchanges which will certainly strengthen the depopulation of rural areas. Besides, although farmers are not aware of that yet, the process of globalisation allows the emergence of new competitors who are also able to produce typical Mediterranean products. According to the author of this paper, the relevant response to the risk of economic deterioration faced by the Mediterranean agriculture is in the definition and the implementation of a common policy encompassing the two banks of the Mediterranean Sea.

\section{INDEX}

Index géographique : Europe, Méditerranée

Mots-clés : politique agricole, libéralisme, commerce agroalimentaire, fruits et légumes 
AUTEUR

JEAN-LOUIS RASTOIN

Professeur à SupAgro, directeur de l'UMR Moisa. 